\title{
Stability of thermoelastic contact for a rectangular elastic block sliding against a rigid wall
}

\author{
M. Ciavarella ${ }^{\mathrm{a}, *}$, J.R. Barber ${ }^{\mathrm{b}}$ \\ ${ }^{a}$ CEMeC - Centre of Excellence in Computational Mechanics, V.le Japigia 182, Politecnico di Bari, 70125 Bari, Italy \\ ${ }^{\mathrm{b}}$ Department of Mechanical Engineering, University of Michigan, Ann Arbor, MI 48109-2125, USA
}

Received 13 November 2003; accepted 5 October 2004

Available online 8 April 2005

\begin{abstract}
In this note, we determine the stability boundary for the thermoelastic contact of a rectangular elastic block sliding against a rigid wall in the presence of a pressure-dependent thermal contact resistance. This geometry can be seen as intermediate between the idealized 'Aldo' rod model and continuum solutions for the elastic half-plane.

The solution is obtained by comparing the expression for the perturbed boundary condition including frictional heating with that for purely static loading, already solved by Yeo and Barber (1995). The critical sliding speed is obtained as a function of the temperature difference imposed between the wall and the free end.

In most cases, frictional heating tends to destabilize the system. However, for certain forms of the resistance-pressure law, the opposite conclusion is reached and the system can be stable for all sliding speeds.
\end{abstract}

(c) 2004 Elsevier SAS. All rights reserved.

Keywords: Thermal contact resistance; TEI; Hot spotting

\section{Introduction}

In thermoelastic contact problems, the temperature distribution causes thermoelastic distortion which influences the contact pressure distribution. If this in turn influences the heat transfer problem, the resulting feedback is potentially unstable, leading to non-uniform temperature and pressure distributions and regions of separation.

Two distinct mechanisms are known which can cause such feedback. If the two bodies slide over each other, frictional heat will be generated proportional to the local contact pressure, leading to the phenomenon known as Frictionally-excited thermoelastic instability or TEI (Barber, 1969; Dow and Burton, 1972). Alternatively, in static contact where there is no frictional heating, thermomechanical coupling can result from the existence at the interface of a thermal contact resistance which depends on the local contact pressure (Barber et al., 1980). There is ample experimental evidence for such a contact resistance (e.g. Clausing and Chao, 1965; Thomas and Probert, 1970) and many authors have developed theoretical models of the process, based on statistical descriptions of the roughness of the contacting bodies (e.g. Shlykov and Ganin, 1964; Cooper et al., 1969).

\footnotetext{
* Corresponding author.

E-mail address: mciava@poliba.lt (M. Ciavarella).

URL: http://cemec.poliba.it/ (M. Ciavarella).
} 
Burton et al. (1973) investigated the TEI problem for two sliding half-planes by determining the condition under which a small perturbation on the uniform pressure solution could grow exponentially in time. Burton's method has since been applied to both categories of thermoelastic stability problem in both analytical and numerical (finite element) form (Lee and Barber, 1993; Yeo and Barber, 1995, 1996; Yi et al., 2000).

Most treatments of the TEI problem assume that perfect thermal contact occurs in the contact area, since this permits a simple condition of temperature continuity to be written at the interface which implicitly defines the partition of frictional heat between the two contacting bodies. However, there is every reason to expect a pressure-dependent thermal contact resistance at the sliding interface (Johansson, 1993) and this introduces the possibility of interaction between the two instability mechanisms. Recent papers have examined the effect of this interaction for one-dimensional rod models (Ciavarella et al., 2003; Afferrante and Ciavarella, 2004a) and on the sliding contact of two half-planes (Afferrante and Ciavarella, 2004b). For a single rod, built in at the non-contacting end, existence of a steady state can only be guaranteed below a certain speed $V_{\infty}$, above which, for some initial conditions, the pressure grows without limit causing seizure (Ciavarella et al., 2003). Similar effects are produced in a shaft rotating inside a concentric cylindrical bushing (Awrejcewicz and Pyryev, 2002). The Aldo model comprises two independent parallel rods sliding against the same rigid surface and joined at the non-contacting ends, where an axial force is applied (Afferrante and Ciavarella, 2004a). In this case, seizure cannot occur because the total axial force is prescribed. The state in which both rods are in contact with equal contact pressures is always possible and it is both unique and stable below a certain sliding speed $V_{u}$. Above this speed, additional steady states are obtained and these can involve one of the rods separating from the wall and/or both rods remaining in contact but with dissimilar contact pressures. The solution with equal contact pressures becomes unstable above some sliding speed $V_{s}$, which may coincide with or exceed $V_{u}$, depending on the system parameters.

Afferrante and Ciavarella (2004b) applied Burton's method to the sliding of a thermoelastic half-plane against a rigid wall with a pressure-dependent contact resistance. They found that for a given wavelength of disturbance, the uniform pressure solution is stable below a certain critical heat flux $q_{\mathrm{cr}}$, but that the value of $q_{\mathrm{cr}}$ can be increased or decreased by sliding, depending on the sign of the function $R_{0}+p_{0} R^{\prime}$, where $R_{0}, p_{0}$ are the thermal contact resistance and the contact pressure in the uniform steady state and $R^{\prime}$ is the corresponding gradient of the resistance/pressure relation.

The Aldo model and the half-plane exhibit significantly different behaviour, even though both geometries are typically used as idealizations of thermoelastic contact for finite continuous bodies. In the present paper, we therefore consider a more realistic intermediate case in which a rectangular block slides against a rigid plane. Relative to the static contact problem, we shall show that the introduction of frictional heating merely introduces an additional pressure-dependent term into the perturbed thermal interface condition and this permits us to write down the stability criterion for the uniform pressure solution, using results for the static contact problem due to Yeo and Barber (1995, 1996).

\section{Yeo's problem with sliding}

We consider the problem illustrated in Fig. 1, in which the rectangular block $0<x<L,-h<y<h$ is in out-of-plane sliding contact with a rigid wall at $x=0$ and loaded by a uniform pressure $p_{0}$ at $x=L$, the other surfaces $y= \pm h$ being traction-free. Thus the mechanical boundary conditions are

$$
\begin{aligned}
& u_{x}(0, y, t)=0 ; \quad \sigma_{x y}(0, y, t)=0 \\
& \sigma_{x x}(L, y, t)=-p_{0} ; \quad \sigma_{x y}(L, y, t)=0
\end{aligned}
$$

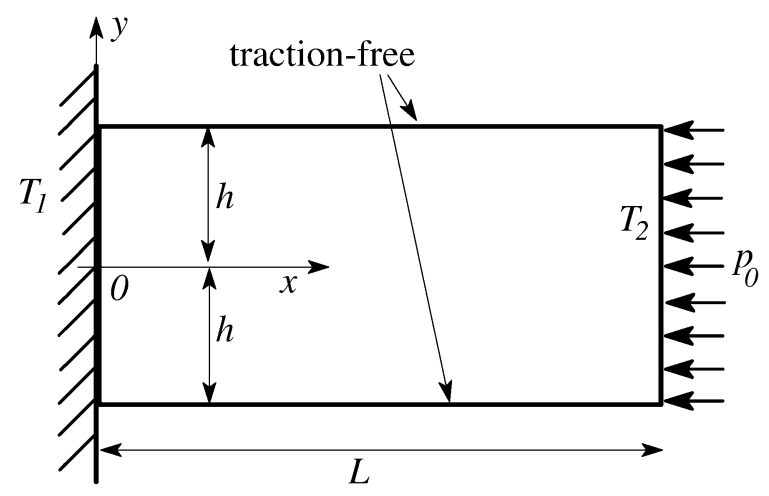

Fig. 1. Rectangular block sliding against a rigid wall. 


$$
\sigma_{y x}(x, \pm h, t)=0 ; \quad \sigma_{y y}(x, \pm h, t)=0,
$$

where $t$ is time.

Frictional dissipation leads to the generation of heat

$$
q(y, t)=f V p(y, t)
$$

at the contact interface, where $V$ is the sliding speed, $f$ is the coefficient of friction and $p(y, t)=-\sigma_{x x}(0, y, t)$ is the contact pressure. We assume the existence of a pressure-dependent thermal contact resistance $R(p)$ between this heat source and the rigid wall, which is assumed to be maintained at uniform temperature $T_{1}$. The heat flow into the wall is therefore

$$
q_{W}=\frac{T(0, y, t)-T_{1}}{R(p)}
$$

and the remaining heat $q_{B}$ must flow into the block, giving

$$
q_{B}=-K \frac{\partial T}{\partial x}(0, y, t)=f V p(y, t)-\frac{T(0, y, t)-T_{1}}{R(p)},
$$

where $K$ is the thermal conductivity of the block material. The other end of the block $x=L$ is maintained at uniform temperature $T_{2}$ and the sides $y= \pm h$ are thermally insulated.

\subsection{The steady-state solution}

Dundurs' theorem (Dundurs, 1974; Barber, 1980) tells us that steady state conduction of heat into the block at $x=0$ will cause a locally convex curvature and this in turn will cause the steady state solution of the thermoelastic contact problem to have a non-uniform pressure with the maximum pressure occurring at the origin. However, sliding will cause local wear which is proportional to contact pressure and this will tend ultimately to equalize the contact pressure. In the presence of wear, the only permissible steady state is one in which a time-independent wear rate leads to a kinematically admissible rigid-body motion. For the symmetrically loaded rectangular block, this requires that the wear rate and hence the steady-state contact pressure be uniform.

In this steady state, we therefore have $p(y, t)=p_{0}$ and the temperature must be a linear function of $x$, giving

$$
\frac{\partial T}{\partial x}=\frac{T_{2}-T_{0}}{L},
$$

where $T_{0}$ is the steady state temperature at $x=0$. Solving Eqs. (4), (5), we have

$$
T_{0}=\frac{L R_{0} f V p_{0}+L T_{1}+K R_{0} T_{2}}{K R_{0}+L} ; \quad q_{0}=\frac{K\left(R_{0} f V p_{0}+T_{1}-T_{2}\right)}{K R_{0}+L},
$$

where $R_{0}=R\left(p_{0}\right)$ and $q_{0}$ is the steady-state value of $q_{B}$.

\subsection{Stability of the steady state}

The stability of this steady state can be analyzed using the technique due to Yeo and Barber (1995, 1996). For this purpose we need to perform a linear perturbation of Eq. (4) about the steady state. Differentiating this equation and denoting the perturbed quantities by tildes, we obtain

$$
-K \frac{\partial \widetilde{T}}{\partial x}(0, y, t)=f V \tilde{p}(y, t)-\frac{\widetilde{T}(0, y, t)}{R_{0}}+\frac{\left(T_{0}-T_{1}\right) R^{\prime} \tilde{p}}{R_{0}^{2}},
$$

where $R^{\prime}$ is the derivative of the resistance function $R(p)$ at the steady-state value $p_{0}$. Using (6) to eliminate $T_{0}$, this equation can be written in the alternative form

$$
-K R_{0} \frac{\partial \widetilde{T}}{\partial x}(0, y, t)=(\beta f V+\gamma) \tilde{p}-\widetilde{T},
$$

where

$$
\beta=R_{0}+\frac{L R^{\prime} p_{0}}{K R_{0}+L} ; \quad \gamma=\frac{K\left(T_{2}-T_{1}\right) R^{\prime}}{K R_{0}+L} .
$$




\subsubsection{Solution with no frictional heating}

In the special case where $V=0$, Eq. (8) reduces to

$$
-K R_{0} \frac{\partial \widetilde{T}}{\partial x}(0, y, t)=\gamma \tilde{p}-\widetilde{T} .
$$

The corresponding stability problem was solved by Yeo and Barber (1996) using a finite element description of the perturbation problem. They showed that instability occurred at a critical value of the dimensionless heat flux

$$
Q^{*}=-E \alpha R^{\prime} q_{0},
$$

where $E, \alpha$ are Young's modulus and the coefficient of thermal expansion respectively for the material of the block and plane strain conditions are assumed. If the aspect ratio

$$
r=\frac{L}{h}>1,
$$

the stability boundary is closely approximated by the solution for the semi-infinite strip. Yeo and Barber (1995) solved the latter problem by expanding the stress and temperature fields as a series of eigenfunctions and found that their converged numerical results could be closely approximated by the simple expression

$$
Q^{*}=2(1-v)\left(1+3.88 R^{*}\right),
$$

where $v$ is Poisson's ratio and

$$
R^{*}=\frac{K R_{0}}{h} .
$$

Substituting (11), (14) into (13) and using (6) to eliminate $q_{0}$, we can write Yeo and Barber's criterion in the form

$$
E \alpha \gamma>2(1-v)\left(1+\frac{3.88 K R_{0}}{h}\right)
$$

for instability.

\subsubsection{Effect of frictional heating}

When $V \neq 0$, the only change in the statement of the stability problem is represented by the replacement of Eq. (10) by (8). Comparing these equations, we see that the coefficient $\gamma$ on $\tilde{p}$ is replaced by $\beta f V+\gamma$. All the other boundary conditions in the two problems are identical, so we can conclude by inspection that the steady state with sliding is unstable if

$$
E \alpha(\beta f V+\gamma)>2(1-v)\left(1+\frac{3.88 K R_{0}}{h}\right) .
$$

\section{Discussion}

In interpreting Eqs. (15), (16), we should note that the contact resistance $R$ generally falls with increasing pressure, so $R^{\prime}<0$. It follows that in the absence of sliding, the system is unstable only for sufficiently large negative values of $\left(T_{2}-T_{1}\right)$, implying that the heat flows from the wall into the block. The first term in (16) will tend to destabilize the system - i.e. to cause instability to occur at a lower negative value of $\left(T_{2}-T_{1}\right)$ - if and only if

$$
\beta>0 .
$$

This criterion reduces to that obtained for the half-plane in the limit where $L \gg K R_{0}$.

The inequality (17) is satisfied for most simple idealizations of the resistance law. For example, it is satisfied for all values of $p_{0}$ if the resistance is inversely proportional to pressure or if it has the more general form

$$
R_{0}=B+\frac{A}{p_{0}} ; \quad A>0, B>0 .
$$

However, plausible resistance functions can be constructed that violate (17) at least in some restricted range of contact pressures. Such cases will arise if there is a rapid reduction of contact resistance at some finite value of contact pressure. Consider for example, the resistance function

$$
R_{0}=\frac{R_{1}}{1+p_{0}^{2} / p_{1}^{2}},
$$


where $R_{1}, p_{1}$ are constants. Substituting into (17) and cancelling non-negative factors, we obtain

$$
\left[L-K R_{1}\right] p_{0}^{2}<\left(K R_{1}+L\right) p_{1}^{2},
$$

which is violated for

$$
p_{0}>p_{\mathrm{cr}} \equiv p_{1} \sqrt{\frac{L+K R_{1}}{L-K R_{1}}}
$$

if $L>K R_{1}$. Frictional heating would then tend to stabilize the system in this range.

A special case of some interest is that in which there is no externally imposed temperature difference, so $\left(T_{2}-T_{1}\right)=0$. The stability criterion (16) then reduces to

$$
E \alpha \beta f V>2(1-v)\left(1+\frac{3.88 K R_{0}}{h}\right)
$$

and we conclude that the system will be stable at all speeds for the resistance law (20) if $p_{0}>p_{\mathrm{cr}}$. For $p_{0}<p_{\mathrm{cr}}$, there will be a critical sliding speed $V_{\mathrm{cr}}$ which is pressure-dependent.

A limiting case where frictional heating would have no effect on the stability of the system can be defined by the condition

$$
\beta \equiv R_{0}+\frac{L R^{\prime} p_{0}}{K R_{0}+L}=0
$$

This defines a linear ordinary differential equation for the function $R_{0}\left(p_{0}\right)$ whose general solution is easily shown to be

$$
R_{0}=\frac{L}{C p_{0}-K},
$$

where $C$ is an arbitrary constant. This relation is clearly physically unrealistic for $p \leqslant K / C$, but for higher pressures it defines a resistance that falls with increasing pressure and as remarked above it is quite conceivable that in some special cases it might approximate the physical law.

\section{Conclusions}

The stability boundary for a rectangular block sliding against a rigid plane with a local pressure-dependent thermal contact resistance has been deduced from results for the corresponding problem without sliding. It is clear that similar relationships could be established for other geometries in which the static stability solution is known.

As in the case of the thermoelastic half-plane, we find that the effect of sliding is generally to reduce the temperature difference required for instability, but if the thermal resistance exhibits a sufficiently rapid decrease with contact pressure in some range, sliding can exert a stabilizing effect. This contrasts with results for the idealized Aldo model, for which instability is always predicted at sufficiently large sliding speed.

In all cases, the critical sliding speed depends on the contact pressure, in contrast to systems in which there is no thermal contact resistance.

\section{References}

Afferrante, L., Ciavarella, M., 2004a. The thermoelastic Aldo contact model with frictional heating. J. Mech. Phys. Solids, in press.

Afferrante, L., Ciavarella, M., 2004b. Frictionally-excited thermoelastic instability in the presence of contact resistance. J. Strain Anal. Engrg. Des. 39, 351-357.

Awrejcewicz, J., Pyryev, Y., 2002. Thermoelastic contact of a rotating shaft with a rigid bush in conditions of bush wear and stick-slip movements. Int. J. Engrg. Sci. 40, 1113-1130.

Barber, J.R., 1969. Thermoelastic instabilities in the sliding of conforming solids. Proc. Roy. Soc. London Ser. A 312, $381-394$.

Barber, J.R., 1980. Some implications of Dundurs' theorem for thermoelastic contact and crack problems. J. Mech. Engrg. Sci. $22,229-232$.

Barber, J.R., Dundurs, J., Comninou, M., 1980. Stability considerations in thermoelastic contact. ASME J. Appl. Mech. 47, 871-874.

Burton, R.A., Nerlikar, V., Kilaparti, S.R., 1973. Thermoelastic instability in a seal-like configuration. Wear 24, 177-188.

Ciavarella, M., Johansson, L., Afferrante, L., Klarbring, A., Barber, J.R., 2003. Interaction of thermal contact resistance and frictional heating in thermoelastic instability. Int. J. Solids Structures 40, 5583-5597.

Clausing, A.M., Chao, B.T., 1965. Thermal contact resistance in a vacuum environment. ASME J. Heat Transfer 87, $243-251$.

Cooper, M.G., Mikic, B.B., Yovanovich, M.M., 1969. Thermal contact conductance. Int. J. Heat Mass Transfer 12, $279-300$.

Dow, T.A., Burton, R.A., 1972. Thermoelastic instability of sliding contact in the absence of wear. Wear 19, 315-328. 
Dundurs, J., 1974. Distortion of a body caused by free thermal expansion. Mech. Res. Commun. 1, 121-124.

Johansson, L., 1993. Model and numerical algorithm for sliding contact between two elastic half-planes with frictional heat generation and wear. Wear 160, 77-93.

Lee, K., Barber, J.R., 1993. Frictionally-excited thermoelastic instability in automotive disk brakes. ASME J. Tribology 115, 607-614.

Shlykov, Yu.P., Ganin, Ye.A., 1964. Thermal resistance of metallic contacts. Int. J. Heat Mass Transfer 7, 921-929.

Thomas, T.R., Probert, S.D., 1970. Thermal contact resistance: the directional effect and other problems. Int. J. Heat Mass Transfer $13,789-807$.

Yeo, T., Barber, J.R., 1995. Stability of a semi-infinite strip in thermoelastic contact with a rigid wall. Int. J. Solids Structures 32, $553-567$.

Yeo, T., Barber, J.R., 1996. Finite element analysis of the stability of static thermoelastic contact. J. Thermal Stresses 19, 169-184.

Yi, Y.-B., Barber, J.R., Zagrodzki, P., 2000. Eigenvalue solution of thermoelastic instability problems using Fourier reduction. Proc. Roy. Soc. London A Ser. 456, 2799-2821. 